\title{
$\mathrm{Ag}-\mathrm{Cu}$ 钎料与双相陶瓷透氧膜的润湿和界面反应研究
}

\author{
张莉莉, 李可, 于称称, 张玉文, 吴成章, 丁伟中 \\ (上海大学 省部共建高品质特殊钢冶金与制备国家重点实验室，上海 200072)
}

摘 要: 用座滴法测试 $\mathrm{Ag}-\mathrm{Cu}$ 合金钎料对 $\mathrm{Ce}_{0.8} \mathrm{Gd}_{0.2} \mathrm{O}_{2-\delta}-\mathrm{NdBaCo}_{2} \mathrm{O}_{5+\delta}(\mathrm{CGO}-\mathrm{NBCO})$ 双相透氧膜的润湿性, 利用 SEM-EDS 分析润湿和界面反应机理。结果表明: 空气条件下 Ag-Cu 合金与 CGO-NBCO 间的润湿遵从界面反应润 湿机制。随着 $\mathrm{Cu}$ 含量的增加, $\mathrm{Ag}-\mathrm{Cu}$ 合金对透氧膜润湿性能提高, $\mathrm{Cu}$ 含量为 $6.6 \mathrm{~mol} \% \sim 15.8 \mathrm{~mol} \%$ 时, 润湿角在 $35^{\circ} \sim 20^{\circ}$ 左右。在润湿界面处出现 $\mathrm{Cu}$ 氧化物的富集, 并且在透氧膜侧生成一层由 $\mathrm{Cu}$ 氧化物和 CGO-NBCO 双相透 氧膜反应产生的 Ba-Cu-O、 $\mathrm{Co}-\mathrm{Cu}-\mathrm{O}$ 和 Nd-Ce-Cu-O 等复杂氧化物相构成的产物层, 新的界面反应层的生成有利于 $\mathrm{Ag}$ 基合金钎料的润湿, 改善了钎料的润湿性能。

关 键 词: $\mathrm{Ag}-\mathrm{Cu}$ 合金; $\mathrm{CGO}-\mathrm{NBCO}$ 双相透氧膜; 润湿; 界面反应

中图分类号: TQ174 文献标识码: A

\section{Wetting Properties and Interface Reaction Mechanism of Ag-Cu Brazes on Dual-phase Membrane Ceramic}

\author{
ZHANG Li-Li, LI Ke,YU Chen-Chen, ZHANG Yu-Wen, WU Cheng-Zhang, DING Wei-Zhong
}

(State Key Laboratory of Advanced Special Steels, Shanghai University, Shanghai 200072, China)

\begin{abstract}
A reactive air brazing Ag-CuO alloy was evaluated for brazing dual phase oxygen membrane $\mathrm{Ce}_{0.8} \mathrm{Gd}_{0.2} \mathrm{O}_{2-\delta^{-}}$ $\mathrm{NdBaCo}_{2} \mathrm{O}_{5+\delta}(\mathrm{CGO}-\mathrm{NBCO})$. Contact angle tests were observed between $\mathrm{Ag}-\mathrm{Cu}$ brazes and CGO-NBCO in air and the interfacial microstructures of the used samples were evaluated by SEM. The results indicate that the wetting angle decreases from $35^{\circ}$ to $20^{\circ}$ with the content of $\mathrm{Cu}$ increased from $6.6 \mathrm{~mol} \%$ to $15.8 \mathrm{~mol} \%$. It is found that there are copper oxide-enriched zones formed in the junction of the alloy and substrate. The wettability between $\mathrm{Ag}-\mathrm{CuO}$ alloy and oxygen membrane CGO-NBCO is improved due to the interfacial reaction between $\mathrm{Cu}$ oxides in braze and the surface layers of CGO-NBCO substrates to form compounds of $\mathrm{Ba}-\mathrm{Cu}-\mathrm{O}, \mathrm{Co}-\mathrm{Cu}-\mathrm{O}$ and $\mathrm{Nd}-\mathrm{Ce}-\mathrm{Cu}-\mathrm{O}$ oxides. The new formed interface reaction layers promote the wettability of the rest braze.
\end{abstract}

Key words: $\mathrm{Ag}-\mathrm{Cu}$ alloy brazing filler; dual-phase membranes ceramic; wettability; interface reaction

富氧燃烧技术采用 $\mathrm{O}_{2} / \mathrm{CO}_{2}$ 作为化石燃料的助 燃剂, 可以消除空气助燃带来的氮化物污染和氮气 分离等问题, 产生高浓度的 $\mathrm{CO}_{2}$ 便于后续的捕捉与 封存, 具有操作便利和高效率特点, 是一种有潜力 地解决 $\mathrm{CO}_{2}$ 温室效应的技术。混合导体透氧膜在高
温下 $\left(\right.$ 一般工作温度为 $800 \sim 950^{\circ} \mathrm{C}$ ) 可以直接从空气 中分离制备纯氧，实现纯氧制备与富氧燃烧过程的 耦合，从而大大地降低投资和过程操作成本，在富 氧燃烧技术方面具有良好的应用前景。实现基于混 合导体透氧膜的富氧燃烧技术，必须开发出能够耐

收稿日期: 2015-11-09; 收到修改稿日期：2015-12-23

基金项目：国家自然科学基金(51174133,51274139); 国家杰出青年科学基金(51225401)

National Natural Science Foundation of China (51174133, 51274139); National Science Fund for Distinguished Young Scholars (51225401)

作者简介: 张莉莉(1991-), 女, 硕士研究生. E-mail: springzlli@163.com

通讯作者: 张玉文, 副研究员. E-mail: springzyw@163.com 
受 $\mathrm{CO}_{2}$ 气氛的透氧膜材料。单相的钙铁矿型 $\left(\mathrm{ABO}_{3}\right)$ 混合氧离子-电子导体透氧膜材料, 其 $\mathrm{A}$ 位主要为碱 土金属, 易与 $\mathrm{CO}_{2}$ 生成碳盐, 在富氧燃烧条件下稳定 性和机械性能极差, 不能满足实际应用要求 ${ }^{[1-2]}$ 。由钙 钛矿一氧离子导体构成的双相混合导体透氧膜, 如 $\mathrm{Ce}_{0.8} \mathrm{Gd}_{0.2} \mathrm{O}_{2-\delta}-\mathrm{NdBaCo}_{2} \mathrm{O}_{5+\delta}$ 在 $\mathrm{CO}_{2}$ 气氛下表现出较 好的稳定性, 具有很大的应用潜力 ${ }^{[3-4]}$ 。

䥻钛矿一氧离子导体构成的双相混合导体透氧 膜材料, 如 $\mathrm{Ce}_{0.8} \mathrm{Gd}_{0.2} \mathrm{O}_{2-\delta}-\mathrm{NdBaCo}_{2} \mathrm{O}_{5+\delta}$ 等在高真空 或氧分压极低的还原气氛下会发生相分解, 传统的 高真空或还原气氛下的活性金属钎焊等方法不适合 陶瓷透氧膜组件化高温封接。为了解决这一问题, Weil 研究组提出了空气反应钎焊方法(Reactive Air Brazing, 简称 $\mathrm{RAB})^{[5]}$, 该方法是在空气下利用贵金 属基合金(如 $\mathrm{Ag}-\mathrm{Cu}$ ) 钎焊透氧膜陶瓷, 得到的封接 件具有较好的抗氧化性能, 并且钎焊过程在空气气 氛下完成, 避免了低氧分压下陶瓷透氧膜材料的分 解问题。Weil 等 ${ }^{[5-6]}$ 采用座滴法研究了空气下 $\mathrm{Ag}-\mathrm{Cu}$ 对 $\mathrm{La}_{0.6} \mathrm{Sr}_{0.4} \mathrm{Co}_{0.2} \mathrm{Fe}_{0.8} \mathrm{O}_{3-\delta}$ 透氧膜的润湿性能, 发现 增加 $\mathrm{Cu}$ 含量有利于改善润湿性能, 当 $\mathrm{Cu}$ 含量在 $1.4 \mathrm{~mol} \% \sim 16 \mathrm{~mol} \%$ 时, 钎焊接头具有较好的润湿性 和连接强度。Dabbarh 等 ${ }^{[7]}$ 采用 $\mathrm{Ag}-\mathrm{Cu}$ 合金空气反 应钎焊 $\mathrm{Ba}_{0.5} \mathrm{Sr}_{0.5} \mathrm{Co}_{0.8} \mathrm{Fe}_{0.2} \mathrm{O}_{3-\delta}$ 透氧膜与不锈钢支撑 体, 发现钎料合金与透氧膜和不锈钢都有较好的润 湿性能, 在两侧连接界面上都发生较严重的组分扩 散和化学反应。本课题组研究了空气下 $\mathrm{Ag}-\mathrm{Cu}$ 钎料 在 $\mathrm{BaCo}_{0.7} \mathrm{Fe}_{0.2} \mathrm{Nb}_{0.1} \mathrm{O}_{3-\delta}$ 透氧膜上的润湿行为, 发现 润湿性能随着钎料中 $\mathrm{Cu}$ 含量增加而提高, 但当 $\mathrm{Cu}$ 含量大于 $8 \mathrm{~mol} \%$ 后, 钎料和透氧膜反应严重, 对透 氧膜件的稳定性影响明显 ${ }^{[8-11]}$ 。本工作利用座滴法 研究了空气中 $\mathrm{Ag}-\mathrm{Cu}$ 合金钎料与新型双相陶瓷透氧 膜 $\mathrm{Ce}_{0.8} \mathrm{Gd}_{0.2} \mathrm{O}_{2-\delta}-\mathrm{NdBaCo}_{2} \mathrm{O}_{5+\delta}$ (简称 CGO-NBCO)的 界面润湿和反应行为, 并结合润湿界面微观结构的测 试分析, 探讨了润湿性能变化和界面连接机理, 以期 为双相陶瓷透氧膜件可靠封接提供理论和技术支持。

\section{1 实验方法}

\section{1 试样的制备}

采用溶胶一凝胶法制备新型双相陶瓷透氧膜所 用的两种复合氧化物 $\mathrm{Ce}_{0.8} \mathrm{Gd}_{0.2} \mathrm{O}_{2-\delta}$ 和 $\mathrm{NdBaCo}_{2} \mathrm{O}_{5+\delta}$ 。 按化学计量系数称取化学纯的 $\mathrm{Ce}\left(\mathrm{NO}_{3}\right)_{3} \cdot 6 \mathrm{H}_{2} \mathrm{O}$ 和 $\mathrm{Gd}_{2} \mathrm{O}_{3}$, 加适量蒸馏水混合, 加硝酸溶解, 加入相应 量络合剂(EDTA 和柠檬酸), 利用氨水调节 $\mathrm{pH}$ 至 $7 \sim 8$ 之间, $90^{\circ} \mathrm{C}$ 水浴蒸发至溶胶状态, 将溶胶状物质 置于烘箱中 $150^{\circ} \mathrm{C}$ 烘干。待溶胶状物质膨胀为海
绵状多孔固体后取出, 将其置于高温电炉中以 $5^{\circ} \mathrm{C} / \mathrm{min}$ 升温速度升到 $350^{\circ} \mathrm{C}$, 保温 $5 \mathrm{~h}$ 使其有机物充分燃烧 干净, 再将所得粉体充分研磨后置于高温电炉中, 以 $5^{\circ} \mathrm{C} / \mathrm{min}$ 升温速度升到 $650^{\circ} \mathrm{C}$, 保温 $5 \mathrm{~h}$ 后随炉冷 却至室温, 得到粉体 $\mathrm{Ce}_{0.8} \mathrm{Gd}_{0.2} \mathrm{O}_{2-\delta}$ 。按同样的方法 制备 $\mathrm{NdBaCo}_{2} \mathrm{O}_{5+\delta}$ 粉体。将上述两种粉体按重量比 (CGO:NBCO=6:4)充分混合研磨, 然后压制成 $\phi 20 \mathrm{~mm} \times$ $1.5 \mathrm{~mm}$ 的膜片, 再经高温烧结 $\left(1100 \sim 1160^{\circ} \mathrm{C}, 6 \sim 8 \mathrm{~h}\right)$ 制得双相陶瓷透氧膜片。采用溶胶一凝胶法制备的双 相陶瓷透氧膜相对密度为 92 , 用 800 目金相砂纸打 磨膜片表面作为座滴法润湿实验的基片。

按表 1 所示钎料成分称取纯 $\mathrm{Ag}$ 和纯 $\mathrm{Cu}$, 在真 空感应炉中熔炼不同成分的 $\mathrm{Ag}-\mathrm{Cu}$ 合金。将熔炼好 的合金加工成 $\phi 3 \mathrm{~mm} \times 3 \mathrm{~mm}$ 的圆柱体, 作为座滴法 润湿实验的钎料合金试样。

\section{2 润湿实验}

高温座滴法是研究润湿性的主要手段之一, 根 据 Young-Dupre 方程, 通过摄取液滴与基片接触的 侧面轮廓图来测量润湿角, 表征润湿性。通过座滴 法来表征空气气氛中不同成分的 $\mathrm{Ag}-\mathrm{Cu}$ 合金在 CGO-NBCO 双相透氧膜上的润湿行为, 具体实验 过程如下: 将加工好的圆柱体合金钎料放在 CGO$\mathrm{NBCO}$ 双相透氧膜片上, 送入电阻加热炉的恒温区, 在空气气氛下以 $6^{\circ} \mathrm{C} / \mathrm{min}$ 速率从室温升至 $1000^{\circ} \mathrm{C}$, 并在 $1000^{\circ} \mathrm{C}$ 保温 $10 \mathrm{~min}$, 然后以 $10^{\circ} \mathrm{C} / \mathrm{min}$ 的速率降 至室温。在实验过程中从座滴合金开始熔化到保温 结束，用摄像仪实时记录不同温度下熔化钎料液滴 在透氧膜基片上铺展的照片, 用 $\mathrm{CANVAS}^{\mathrm{TM}}$ 软件分 析钎料在透氧膜片上的润湿角。

\section{3 试样测试}

做完润湿实验的样品经镶嵌和界面打磨抛光制 成金相试样，用扫描电镜(SEM, HITACHISU-1500) 对其界面进行微观结构分析, 并结合能谱(EDS)进 行微区元素分布分析。

\section{2 结果与讨论}

\section{$2.1 \mathrm{Ag}$ 基钎料与双相膜的润湿性能}

图 1 为 CGO-NBCO 的 XRD 图谱, 由图谱可以

表 1 Ag-Cu 钎料成分 $/ \mathrm{mol} \%$

Table 1 Composition of Ag-Cu brazes/mol\%

\begin{tabular}{ccc}
\hline Braze & $\mathrm{Cu}$ & $\mathrm{Ag}$ \\
\hline $\mathrm{Ag}$ & 0 & 100 \\
$\mathrm{Ag}-6.6 \mathrm{Cu}$ & 6.6 & 93.4 \\
$\mathrm{Ag}-11.0 \mathrm{Cu}$ & 11.0 & 89.0 \\
$\mathrm{Ag}-15.8 \mathrm{Cu}$ & 15.8 & 84.2 \\
\hline
\end{tabular}




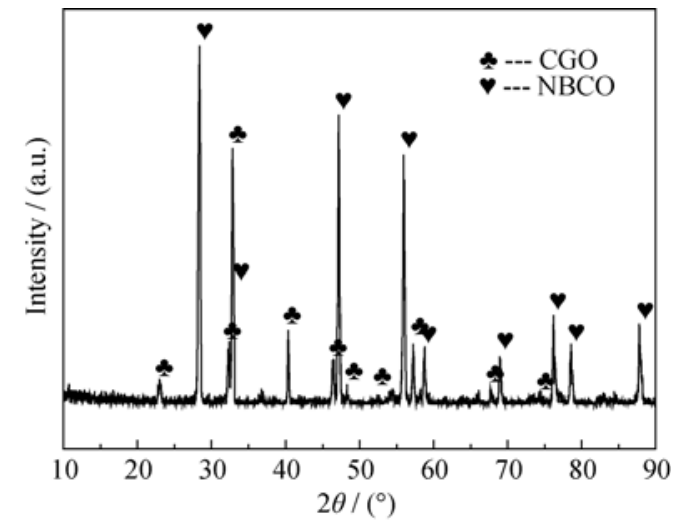

图 1 CGO-NBCO 的 XRD 图谱

Fig. 1 XRD pattern of CGO-NBCO

看出衍射峰分别对应 CGO 和 NBCO 的特征衍射峰, 没有出现明显杂峰, 说明两者之间相容性较好。不 同 $\mathrm{Cu}$ 含量的 $\mathrm{Ag}$ 基合金钎料与 $\mathrm{CGO}-\mathrm{NBCO}$ 双相透 氧膜的润湿角度随温度的变化如图 2 所示。从图 2 可知, 在空气气氛下, 纯 $\mathrm{Ag}$ 对 $\mathrm{CGO}-\mathrm{NBCO}$ 双相膜 不润湿, $965^{\circ} \mathrm{C}$ 和 $1000^{\circ} \mathrm{C}$ 时润湿角分别为 $150^{\circ}$ 和 $140^{\circ}$, 纯 $\mathrm{Ag}$ 熔化后随着温度的升高, 润湿角基本 无变化。向 $\mathrm{Ag}$ 中添加少量的 $\mathrm{Cu}$ 后, 钎料对 CGO$\mathrm{NBCO}$ 双相膜润湿, 并且随着 $\mathrm{Cu}$ 含量的增加, 钎料 的润湿性能越好。 $1000^{\circ} \mathrm{C}$ 下, 钎料中 $\mathrm{Cu}$ 含量由 $6.6 \mathrm{~mol} \%$ 增大到 $15.8 \mathrm{~mol} \%$ 时, 钎料对双相透氧膜的 润湿角由 $35^{\circ}$ 减小到 $20^{\circ}$ 。对于相同 $\mathrm{Cu}$ 含量的 $\mathrm{Ag}$ 基钎料, 钎料熔化后, 随着温度的升高, $\mathrm{Ag}-\mathrm{Cu}$ 合金 与双相透氧膜的润湿角先迅速减小, 当温度达到 $970^{\circ} \mathrm{C}$ 以上, 润湿角基本不再变化。
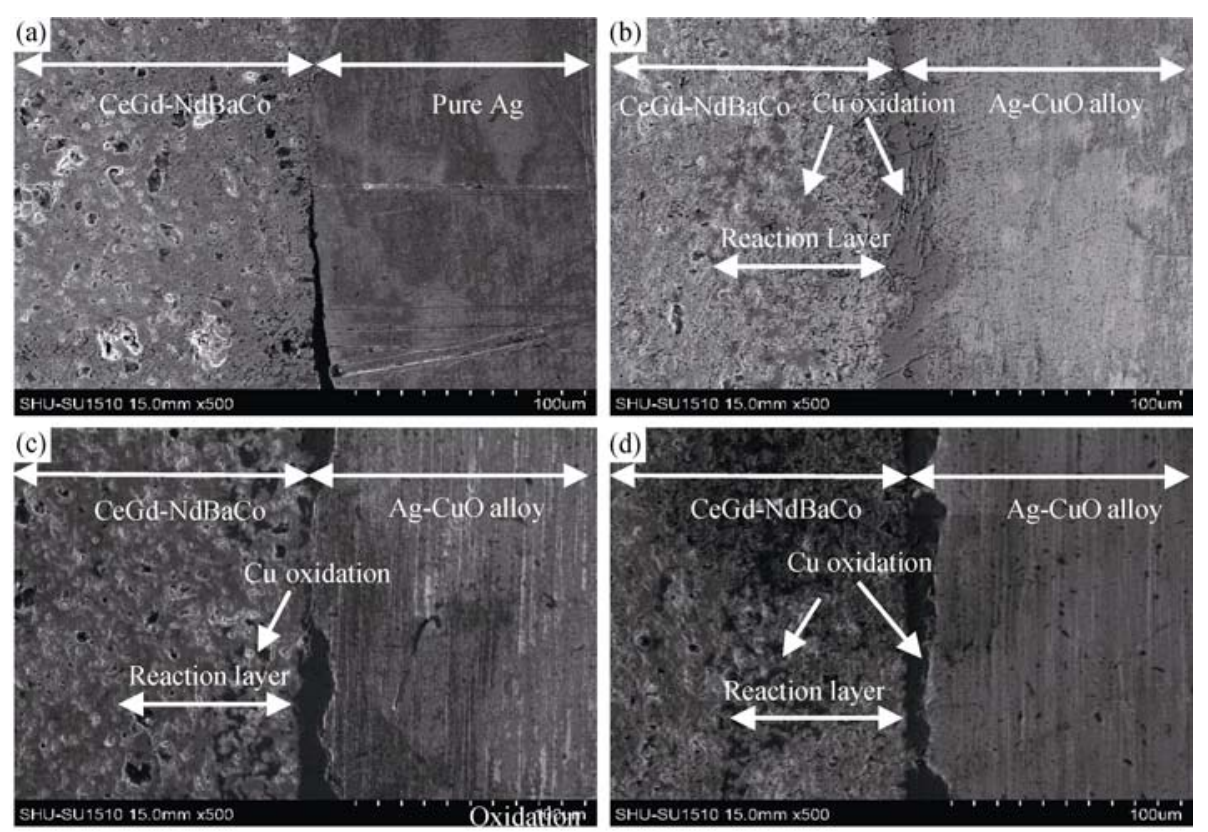

图 $3 \mathrm{Ag}-\mathrm{Cu}$ 钎料与 $\mathrm{CGO}-\mathrm{NBCO}$ 双相透氧膜间润湿界面形貌

Fig. 3 Morphologies of the interfaces between Ag-Cu brazes and CGO-NBCO membrane after wetting experiments (a) $\mathrm{Ag}$; (b) $\mathrm{Ag}-6.6 \mathrm{Cu}$; (c) $\mathrm{Ag}-11 \mathrm{Cu}$; (d) $\mathrm{Ag}-15.8 \mathrm{Cu}$

图 2 不同温度下 $\mathrm{Ag}-\mathrm{Cu}$ 合金与 $\mathrm{CGO}-\mathrm{NBCO}$ 的润湿角

Fig. 2 Contact angles between $\mathrm{Ag}-\mathrm{Cu}$ and $\mathrm{CGO}-\mathrm{NBCO}$ as a function of temperature

刘蛟等 ${ }^{[12]}$ 研究了空气中不同成分 $\mathrm{Ag}-\mathrm{Cu}$ 合金在 $\mathrm{BaCo}_{0.7} \mathrm{Fe}_{0.2} \mathrm{Nb}_{0.1} \mathrm{O}_{3-\delta}(\mathrm{BCFNO})$ 单一钻钛矿相透氧膜上 润湿性能发现, $1000^{\circ} \mathrm{C}$ 下 $\mathrm{Cu}$ 含量分别为 $6.6 \mathrm{~mol} \%$ 、 $11.0 \mathrm{~mol} \%$ 和 $15.8 \mathrm{~mol} \%$ 的钎料合金在 BCFN 透氧膜上 的润湿角分别为 $47^{\circ} 、 40^{\circ}$ 和 $33^{\circ}$ 。在相同温度下, 本 工作相同 $\mathrm{Cu}$ 含量钎料合金在 CGO- NBCO 双相透 氧膜上的润湿角分别为 $35^{\circ} 、 30^{\circ}$ 和 $20^{\circ}$, 润湿角更 小。可见随着合金中 $\mathrm{Cu}$ 含量的增加, 钎料合金与单 相透氧膜、双相透氧膜的润湿角都减小, 即润湿性 能变好。

\section{2 润湿试样的 SEM-EDS 表征}

图 3 为润湿实验后不同 $\mathrm{Ag}$ 基合金钎料与 CGO$\mathrm{NBCO}$ 双相透氧膜之间的界面 SEM 照片。从图 3(a) 中可以看出, 纯 $\mathrm{Ag}$ 钎料与透氧膜的连接界面清晰, 
没有出现反应层, 纯 $\mathrm{Ag}$ 与双相透氧膜的结合很差, 部分区域存在间隙。从图 3(b) (d) 可知, 在 $\mathrm{Ag}$ 中添 加 $\mathrm{Cu}$ 之后, $\mathrm{Ag}-\mathrm{Cu}$ 合金钎料与透氧膜连接界面处存 在一个反应层。EDS 测试结果显示钎料与透氧膜连 接界面上形成的不连续和连续的黑色微区主要是 $\mathrm{Cu}$ 的氧化物。钎料合金中 $\mathrm{Cu}$ 含量较低(如 $6.6 \mathrm{~mol} \%$ ) 时, $\mathrm{Cu}$ 氧化物以小颗粒比较均匀地弥散分布在连接 界面上; 当钎料中的 $\mathrm{Cu}$ 含量升高到 $11 \mathrm{~mol} \%$ 时, $\mathrm{Cu}$ 氧化聚集成带状分布, 但并未完全覆盖连接界面; 当 $\mathrm{Cu}$ 含量进一步提高为 $15.8 \mathrm{~mol} \%$ 时, 在连接界面 上形成一个连续的 $\mathrm{Cu}$ 氧化物聚集层。另外, 从图 3(b) (d) 还可以发现, 在连接界面处透氧膜一侧存 在一个明显反应层, 结合 EDS 检测知道反应层中有 大量的 $\mathrm{Cu}$ 氧化物弥散分布在双相膜基体中。与空 气下 $\mathrm{Ag}-\mathrm{Cu}$ 合金在 BCFNO 透氧膜上的界面反应规 律类似 ${ }^{[12]}$, 随着钎料合金中 $\mathrm{Cu}$ 含量的增加, 界面 反应层厚度不断增大，但在相同温度和钎料合金条 件下, 钎料与 CGO-NBCO 双相透氧膜的界面反应 层厚度比 BCFNO 单相膜更小。

图 4(a) (c)为空气中不同 $\mathrm{Ag}-\mathrm{Cu}$ 合金钎料与 CGO-NBCO 双相膜润湿试样三相界面(三相: 双相 透氧膜 $-\mathrm{Ag}-\mathrm{Cu}$ 合金钎料-空气) 附近截面的 SEMEDS 测试结果。从透氧膜和钎料合金中不同元素面 扫描分布情况(图 4(a) (c)) 可以看出, 在三相界面反 应层中主要元素包括 $\mathrm{Cu} 、 \mathrm{Ba} 、 \mathrm{Co} 、 \mathrm{Ce} 、 \mathrm{Nd}$ 和 $\mathrm{O}$ 。 反应层形成主要是因为 $\mathrm{Cu}$ 元素的扩散和少量 $\mathrm{Ag}$ 的 扩散。合金和透氧膜中没有发现单质 $\mathrm{Cu}$, 说明 $\mathrm{Cu}$ 在空气气氛下润湿透氧膜的过程中全部氧化为 $\mathrm{Cu}$ 的氧化物, 从 $\mathrm{Cu}$ 和 $\mathrm{O}$ 元素分布也可以看出, $\mathrm{Cu}$ 以 氧化物的形式存在, 界面处富 $\mathrm{Cu}$ 带主要是 $\mathrm{Cu}$ 的氧 化物富集。从 $\mathrm{Cu}$ 元素分布可以看出润湿反应结束 后出现的反应区, 主要以 $\mathrm{Cu}$ 的氧化物为主, 同时伴 随有 $\mathrm{Ba} 、 \mathrm{Co} 、 \mathrm{Ce} 、 \mathrm{Nd}$ 的富集, 由 $\mathrm{O}$ 元素的分布图 可以推测, $\mathrm{Ba} 、 \mathrm{Co} 、 \mathrm{Ce} 、 \mathrm{Nd}$ 元素应以氧化物的形式 存在。根据 $\mathrm{BaO}-\mathrm{CuO}_{x}$ 相图可以知道, $\mathrm{BaO}$ 和 $\mathrm{CuO}$ 体系能在 $900^{\circ} \mathrm{C}$ 左右生成低熔点的 $\mathrm{Ba}-\mathrm{Cu}-\mathrm{O}$ 复杂氧 化物 ${ }^{[13]}$ 。查相图可知 $\mathrm{Cu}$ 氧化物与 $\mathrm{Co}$ 氧化物及 $\mathrm{Cu}$ 氧化物、 $\mathrm{Ce}$ 氧化物与 $\mathrm{Nd}$ 氧化物之间也可能会生成 复杂化合物。因此可以推断含 $\mathrm{Cu}$ 的 $\mathrm{Ag}$ 基合金在 CGO-NBCO 双相透氧膜上的润湿过程可能为: (1) $\mathrm{Ag}$ 基合金中的 $\mathrm{Cu}$ 在空气气氛中加热时先氧化成 $\mathrm{Cu}$ 氧化物; (2)钎料高温熔化后 $\mathrm{Cu}$ 氧化物与 CGO$\mathrm{NBCO}$ 双相透氧膜发生反应, 生成 $\mathrm{Ba}-\mathrm{Cu}-\mathrm{O} 、 \mathrm{Co}-\mathrm{Cu}-$ $\mathrm{O}$ 和 $\mathrm{Nd}-\mathrm{Ce}-\mathrm{Cu}-\mathrm{O}$ 等复杂氧化物组成界面反应产物; (3)后续的 $\mathrm{Ag}$ 基钎料合金在生成的界面反应产物上
铺展, 形成最终的润湿钎焊连接状态。

不同温度下 $\mathrm{Ag}-\mathrm{Cu}$ 合金与 $\mathrm{CGO}-\mathrm{NBCO}$ 双相透 氧膜的润湿角随着过程升温的变化趋势(图 2)可以 看出, $\mathrm{Ag}-\mathrm{Cu}$ 合金在熔化后与双相透氧膜存在一个 润湿角逐渐减小的铺展过程, 当温度升至 $970^{\circ} \mathrm{C}$ 以 上, 润湿角随着温度升高基本不再变化, 这一现象 可以用 $\mathrm{Ag}-\mathrm{CuO}_{x}$ 相图加以解释。由前述分析可知, $\mathrm{Ag}$ 基合金中的 $\mathrm{Cu}$ 在空气气氛中加热时先氧化成 $\mathrm{Cu}$ 氧化物, 所以放置在双相透氧膜表面的 $\mathrm{Ag}-\mathrm{Cu}$ 合金 试样熔化润湿过程实际上是 $\mathrm{Ag}-\mathrm{CuO}_{\mathrm{x}}$ 合金的熔化润 湿。 $\mathrm{Ag}-\mathrm{CuO}_{x}$ 相图如图 5 所示, 本研究 $\mathrm{Ag}-\mathrm{Cu}$ 合金 成分选取如图 5 中实线所示 $(\mathrm{A}: \mathrm{Ag}-6.6 \mathrm{Cu}, \mathrm{B}: \mathrm{Ag}$ $11 \mathrm{Cu}, \mathrm{C}: \mathrm{Ag}-15.8 \mathrm{Cu}$ )。依据图 5 所示的 $\mathrm{Ag}-\mathrm{CuO}_{x}$ 相图, 平衡态条件下, 随温度的升高, $\mathrm{Ag}-\mathrm{CuO}_{x}$ 体系在 932 964 ${ }^{\circ} \mathrm{C}$ 的温度区间处于 $\mathrm{CuO}+\mathrm{L}_{2}$ 两相区 $\left(\mathrm{L}_{2}\right.$ 为富 $\mathrm{Ag}$ 相), 绝大部分的 $\mathrm{CuO}$ 以固态存在; 当温度升高 到 $964^{\circ} \mathrm{C}$ 以上后, 体系处于 $\mathrm{L}_{1}+\mathrm{L}_{2}$ 两个液相混合区 $\left(\mathrm{L}_{1}\right.$ 为富 $\mathrm{CuO}_{x}$ 相)。由图 2 所示, 不同的 $\mathrm{Ag}-\mathrm{Cu}$ 合金从开 始熔化到 $970^{\circ} \mathrm{C}$ 左右, 润湿角呈现逐渐减小的过程, 这个区间对应着 $\mathrm{CuO}+\mathrm{L}_{2}$ 两相区，随着温度的升高， 固态的 $\mathrm{CuO}$ 相逐渐减少, 液态钎料中的 $\mathrm{CuO}_{x}$ 相逐渐 增多, 并与 CGO-NBCO 双相透氧膜表面不断发生反 应、铺展, 润湿角逐渐减小, 宏观上表现为润湿性能 逐渐变好。当实验温度升高到 $970^{\circ} \mathrm{C}$ 以上时, 钎料以不 混溶的 $\mathrm{L}_{1}+\mathrm{L}_{2}$ 存在, 在本实验所用的 $\mathrm{CuO}$ 含量 $6.6 \mathrm{~mol} \%$ 到 $15.8 \mathrm{~mol} \%$ 范围内, 根据临界润湿理论可知, 多组分 液体的润湿行为在温度高于某点时将会发生转变, 此 时多组分液体将会出现液液不混溶现象, 即当液体升 高至此温度时, 生成两种不混溶的液相 $\mathrm{L}_{1}+\mathrm{L}_{2}$, 其中 只有一种液相能润湿邻近的固态基体。对于 $\mathrm{Ag}-\mathrm{CuO}_{x}$ 合金，富 $\mathrm{CuO}$ 的液相 $\mathrm{L}_{1}$ 容易润湿固态陶瓷基体，由于 $\mathrm{L}_{1}$ 容易润湿固态陶瓷基体, 因此它会包裹着第二相液 相进入连接界面处, 所以在界面反应区看到少量的富 $\mathrm{Ag}$ 相的存在(如图 4 中 $\mathrm{Ag}$ 元素的面扫描图所示)。由 图 3 和图 4 的润湿试样界面的 SEM 和 EDS 测试结果 可知, 富 $\mathrm{CuO}$ 的 $\mathrm{L}_{1}$ 相起到预润湿的根本原因是 $\mathrm{L}_{1}$ 相 中 $\mathrm{CuO}_{x}$ 与 $\mathrm{CGO}-\mathrm{NBCO}$ 双相透氧膜基体发生反应生成 新的界面反应层, 有利于剩余钎料的润湿。图 3 中不 同试样的微区元素面扫描表明反应层中生成的新物 相可能包括 Ba-Cu-O、Co-Cu-O 和 Nd-Ce-Cu-O 复杂 氧化物。

\section{3 结论}

1) 纯 Ag 对 CGO-NBCO 双相膜不润湿, $\mathrm{Cu}$ 含 

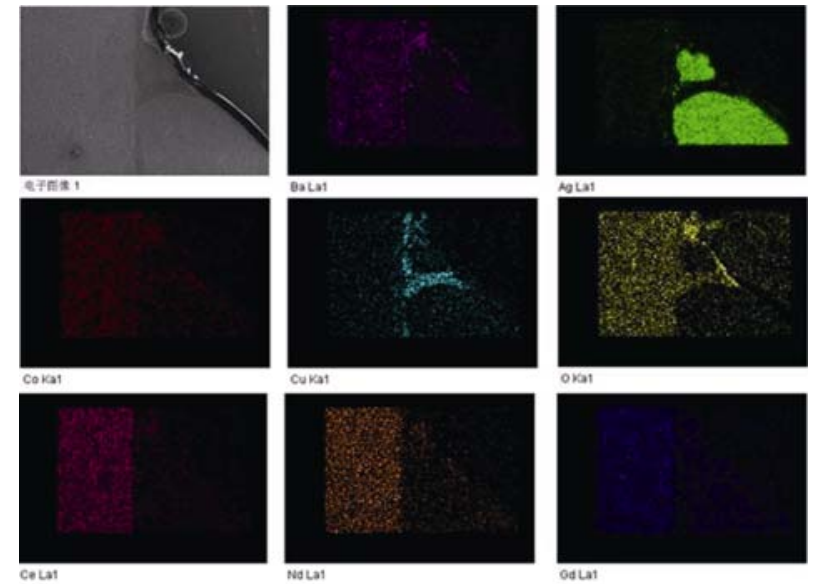

(a) $\mathrm{Ag}-6.6 \mathrm{Cu}$
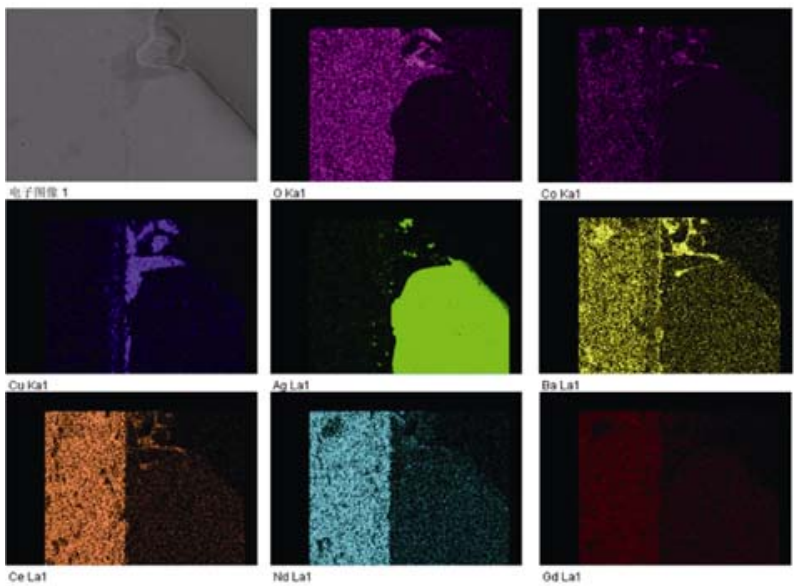

(b) $\mathrm{Ag}-11 \mathrm{Cu}$
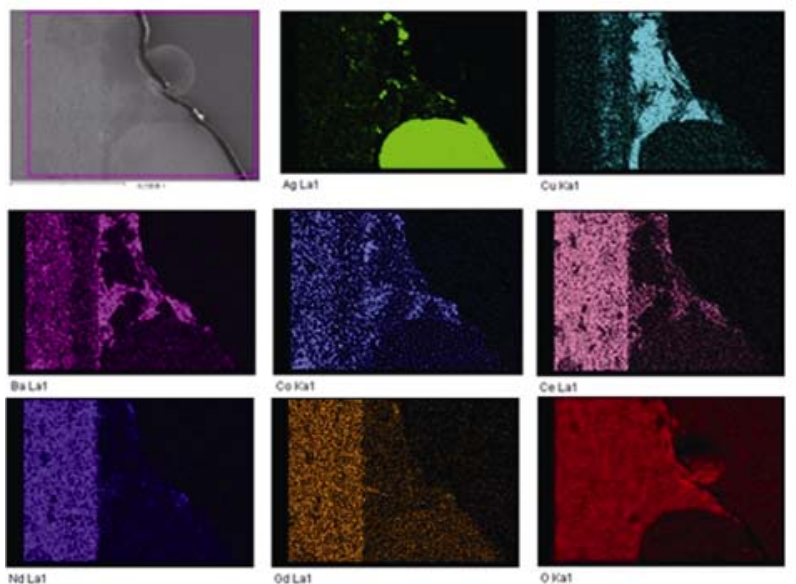

(c) $\mathrm{Ag}-15.8 \mathrm{Cu}$

图 4 不同 $\mathrm{Ag}-\mathrm{Cu}$ 钎料与 $\mathrm{CGO}-\mathrm{NBCO}$ 双相膜润湿界面处面扫 描能谱分析

Fig. 4 EDS analyses of the interface between $\mathrm{Ag}$-Cu brazes and CGO-NBCO membrane

量分别为 $6.6 \mathrm{~mol} \% 、 11.0 \mathrm{~mol} \%$ 和 $15.8 \mathrm{~mol} \%$ 的 $\mathrm{Ag}$ 基 合金钎料对 CGO-NBCO 双相透氧膜润湿良好, 润 湿遵从界面反应润湿机制。

2) 空气气氛下 $\mathrm{Ag}-\mathrm{Cu}$ 合金中 $\mathrm{Cu}$ 在高温加热

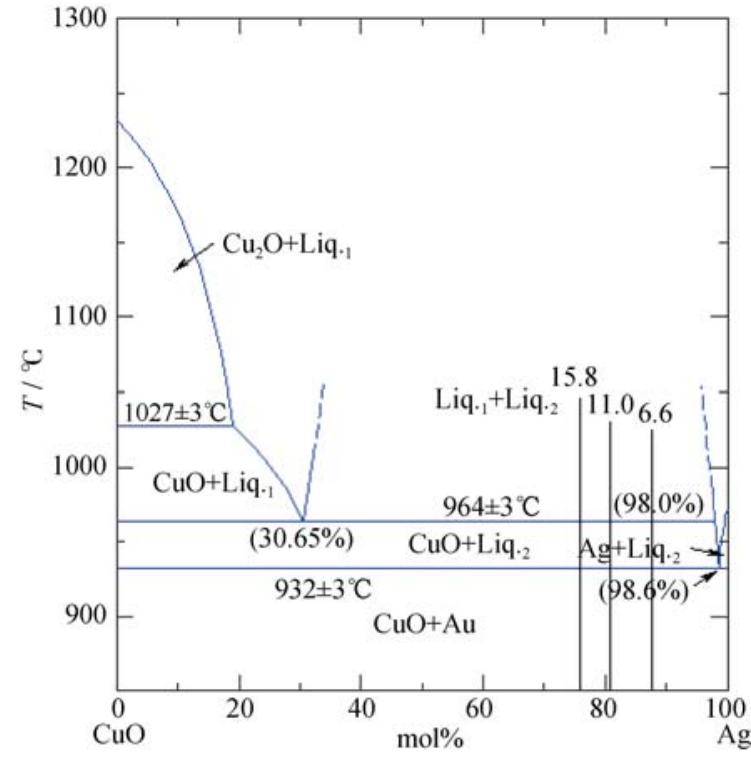

图 $5 \mathrm{Ag}-\mathrm{CuO}$ 相图 ${ }^{[14]}$

Fig. 5 Pseudobinary Ag-CuO phase diagram ${ }^{[14]}$

过程中先被氧化成 $\mathrm{Cu}$ 的氧化物, 合金开始熔化后, $\mathrm{Ag}$ 基合金钎料中 $\mathrm{Cu}$ 氧化物与 $\mathrm{CGO}-\mathrm{NBCO}$ 双相透氧膜 发生反应，生成由复杂氧化物 $\mathrm{Ba}-\mathrm{Cu}-\mathrm{O} 、 \mathrm{Co}-\mathrm{Cu}-\mathrm{O}$ 和 $\mathrm{Nd}-\mathrm{Ce}-\mathrm{Cu}-\mathrm{O}$ 等构成的界面反应层, 新的界面反应层 有利于后续钎料合金的润湿，改善了 $\mathrm{Ag}$ 基合金钎料 对 CGO-NBCO 双相透氧膜的润湿性能。

\section{参考文献:}

[1] WEIL K S, HARDY J S, RICE J P, et al. Brazing as a means of sealing ceramic membranes for use in advanced coal gasification processes. Fuel, 2006, 85(2): 156-162.

[2] SHAO ZONG-PING, XIONG GUO-XING. Mixed conducting oxygen permeative membranes and their application in partial oxidation of methane to syngas process. Process in Chemical, 1999, 11(1): $30-40$.

[3] TONG JIAN-HUA, YANG WEI-SHEN. Methods for selection perovskite-type oxides used as mixed-conducting membranes for oxygen separation. Membrane Science and Technology, 2003, 23(1): 33-42.

[4] CHUANGANG FAN, WEI LIU, ZENGQIANG DENG, et al. Mechanical stability and transport properties of the Sn-promoted $\mathrm{SrCo}_{0.8} \mathrm{Fe}_{0.2} \mathrm{O}_{3-\delta}$ ceramic membrane. Journal of Membrane Science, 2007, 290(1/2): 73-77.

[5] HARDY J S, KIM J Y, WEIL K S. Joining mixed conducting oxides using an air-fired electrically conductive braze. Journal of the Electrochemical Society, 2004, 151(8): 43-49.

[6] WEIL K S, KIMJ Y, HARDY J S. Interfacial analysis of $\left(\mathrm{La}_{0.6} \mathrm{Sr}_{0.4}\right)$ $\left(\mathrm{Co}_{0.2} \mathrm{Fe}_{0.8}\right) \mathrm{O}_{3-\delta}$ substrates wetted by Ag-CuO. Journal of Material 
Science, 2005(40): 2341-2348.

[7] DABBARH S, PFAFF A, ZIOMBRA A, et al. Brazing of mice ceramics to high temperature metals. Ceramic Transactions, 2010 (215): 213-223.

[8] WANG FANG, ZHANG YU-WEN, DING WEI-ZHONG, et al. Pure $\mathrm{Cu}$ braze for sealing oxygen-permeable membrane ceramics and stainless steel support. Journal of Functional Materials, 2010, 41(9): 1540-1542.

[9] WANG FANG, LIU YONG, LU XION-GANG, et al. Effect of $\mathrm{Cu}$ content on interface microstructures of oxygen-permeable membrane brazed with $\mathrm{Ag}-\mathrm{Cu}$ brazing filler. Rare Meterials and Engineering, 2011, 40(10): 1832-1835.

[10] LIU JIAO, ZHANG YU-WEN, LIU XU, et al. The effect of temperature on the interface reaction and connectivity between $\mathrm{Ag}-\mathrm{Cu}$ alloy braze and ceramic oxygen-permeable membrane. Journal of Functional Materials, 2011, 42(2): 313-317.

[11] ZHANG QI-FEI, GENG ZHEN, ZHANG YU-WEN, et al. The wetting and interface reaction mechanism of $\mathrm{Ag}-\mathrm{Cu}-\mathrm{O}$ brazes on $\mathrm{BaCo}_{0.7} \mathrm{Fe}_{0.2} \mathrm{Nb}_{0.1} \mathrm{O}_{3-\delta} \quad$ oxygen-permeable membrane ceramic. Journal of Functional Materials, 2012, 43(1): 77-80.

[12] 刘 蛟. $\mathrm{Ag}-\mathrm{Cu}$ 钎料与透氧膜陶瓷的润湿及界面反应研究. 上海: 上海大学硕士学位论文, 2013.

[13] NEVRIVA M, KRAUS H, SEDMIDUBSKY D. Phase equilibria study in the partially open $\mathrm{Cu}-(\mathrm{O})$ and $\mathrm{Me}-\mathrm{Cu}-(\mathrm{O})(\mathrm{Me}=\mathrm{Ba}, \mathrm{Bi}$, Sr) systems. Thermochim Acta, 1995(282/283): 205- 224.

[14] SHAO Z B, LIU K R, LIU Q L, et al. Eauilibrium phase diagrams in the systems $\mathrm{PbO}-\mathrm{Ag}$ and $\mathrm{CuO}-\mathrm{Ag}$. Journal of the American Ceramic Society, 1993, 76(10): 2663-2664. 